\title{
Reflection and transmission of waves in surface-disordered waveguides
}

\author{
J. A. Sánchez-Gil \\ Instituto de Estructura de la Materia, Consejo Superior de Investigaciones Científicas, Serrano 121, 28006 Madrid, Spain \\ V. Freilikher \\ The Jack and Pearl Resnick Institute of Advanced Technology, Department of Physics, Bar-Ilan University, Ramat-Gan 52900, Israel \\ A. A. Maradudin \\ Department of Physics and Astronomy and Institute for Surface and Interface Science, University of California, Irvine, California 92697 \\ I. V. Yurkevich \\ School of Physics and Space Research, University of Birmingham, Edgbaston, Birmingham B15 2TT, United Kingdom
}

(Received 7 July 1998)

\begin{abstract}
The reflection and transmission amplitudes of waves in disordered multimode waveguides are studied by means of numerical simulations based on the invariant embedding equations. In particular, we analyze the influence of surface-type disorder on the behavior of the ensemble average and fluctuations of the reflection and transmission coefficients, reflectance, transmittance, and conductance. Our results show anomalous effects stemming from the combination of mode dispersion and rough-surface scattering: For a given waveguide length, the larger the mode transverse momentum is, the more strongly is the mode scattered. These effects manifest themselves in the mode selectivity of the transmission coefficients, anomalous backscattering enhancement, and speckle pattern both in reflection and transmission, reflectance and transmittance, and also in the conductance and its universal fluctuations. It is shown that, in contrast to volume impurities, surface scattering in quasi-one-dimensional structures (waveguides) gives rise to the coexistence of the ballistic, diffusive, and localized regimes within the same sample. [S0163-1829(99)09103-1]
\end{abstract}

\section{INTRODUCTION}

The statistical properties of disordered systems is a rich and long-standing problem that attracts many efforts both theoretical and experimental. In quantum solid-state physics much attention is paid to statistics of eigenfunctions and eigenvalues of closed disordered systems. Disorder in solidstate problems is usually represented by impurities that are randomly distributed over the whole sample. For this sort of mesoscopic samples with "bulk disorder" the number of well-established statistical characteristics is enormous (see, for example, Ref. 1, and references therein). The success of mesoscopics may be ascribed to the existence of the nonlinear $\sigma$ model put forward by Efetov. ${ }^{2}$ Although it is a remarkable tool for studying mesoscopic effects, the $\sigma$ model has, however, restricted validity. For example, the system size must be much greater than the mean free path. Generalization to chaotic ballistic systems (i.e., quantum billiards) has recently become a topic of great interest. Progress in this direction has become possible due to the recently proposed field theory for quantum ballistics. ${ }^{3}$ By exploiting the tool, the authors of Refs. 4 and 5 managed to treat different correlators in a clean system within an extremely chaotic limit, when the typical relaxation time is of the order of the flight time (diffusive boundary scattering). They found that naive substitution of the mean free path for the system size into correlators obtained for bulk disorder would give wrong results for the ballistic case, and that, in fact, systems with bulk and surface disorder are not equivalent.
In this paper we address the problem of a ballistic system that is disordered in the sense that there are no bulk scatterers, and the only place where scattering occurs is at a rough boundary. We choose to explore not the statistics of the eigenvalues of completely closed systems (like resonators or quantum dots), but the statistical properties of scattering and reflecting amplitudes in bounded, but open in one direction, systems (waveguiding structures). The key entities for this problem are the transmission and reflection amplitudes. More specifically, we consider a $N$-mode waveguide with the boundary corrugated within a finite interval $(L)$, and study the statistical properties of the transmission through, and reflection from, the disordered segment of the waveguide. This problem arises naturally in the characterization of transport properties related to, for instance, optical waveguides and fibers, remote sensing, radio wave propagation, sonar, shallow water waves, and geophysical probing. ${ }^{6,7}$ On the other hand, it describes as well the electronic transport in mesoscopic systems, ${ }^{8,9}$ being especially relevant to conductance in nanowires. ${ }^{10-13}$

The analogous problem with bulk scatterers was addressed by many authors (see, for example, Refs. 6, 8, 14, and references therein). For a waveguide with bulk disorder all transmission coefficients $T_{m n}$ (subindexes $n$ and $m$ stand for the number of the incident and transmitted mode, respectively) behave in a similar way. Due to the strong intermode mixing all information about the $n$ dependence is washed out after a few scattering events, which means that for $L$ of the order of the scattering length $l$ (and larger) all modes cross over from the ballistic regime to diffusion, and all channels 
become identical. As a result, there exists only one characteristic length scale for all transmittances, the so-called localization length, that is believed to be equal to $\mathrm{Nl}$. In the case $L \ll N l$, each $T_{m n}$ obeys Rayleigh statistics; as the length $L$ increases, all channels (modes) undergo the same changes, and at $L \gg N l$ the crossover to the log-normal distribution (typical for the localized regime) takes place. ${ }^{15-21}$ It might seem that the only distinction of the problem with a rough surface from that with bulk disorder is that the scattering process takes place in a reduced effective volume, which should lead just to a decrease of the mode mixing rate. Naive considerations would suggest that if we introduced a new localization length (which obviously must be much longer than that for the bulk scattering), all results well known for the "bulk" problem should be valid for the "surface" case after proper rescaling. However, the situation is different and much more complicated.

The goal of the present paper is to study the statistical properties of waves transmitted through and reflected from a waveguide with rough boundaries. The length dependences of the reflection and transmission amplitudes for each realization of the surface profile are numerically obtained by solving a system of linear differential equations based on the invariant embedding equations. ${ }^{22}$ Then we calculate the ensemble average and fluctuations of the reflection and transmission coefficients, reflectance, transmittance, and conductance. It is shown in this paper that the interplay between mode dispersion and surface scattering gives rise to many unusual (at least from the point of view of the intuition gained from studies of the volume scattering) effects; one of such effects, the coexistence of different transport regimes at a certain length scale, has been previously reported. ${ }^{23}$

This paper is organized as follows. In Sec. II the theoretical formulation leading to the invariant embedding equations for the matrices of the reflection and transmission amplitudes is developed. The description of the numerical implementation of those equations for the particular waveguide geometry chosen here is detailed in Sec. III. The results thus obtained for the average and fluctuations of the reflection and transmission coefficients are presented and discussed in Sec. IV, whereas those for the total reflection and transmission, and conductance, appear in Sec. V. The relevant conclusions derived from this paper are summarized in Sec. VI.

\section{THEORY}

We start from the wave equation

$$
\left(\Delta+k^{2}\right) \Psi(\mathbf{R})=0,
$$

with the boundary conditions

$$
\Psi\left(\mathbf{R}=\mathbf{R}_{\mathbf{s}}\right)=\left\{\begin{array}{l}
0 \quad \text { for } x<0 \text { and } x>L \\
-\xi(\mathbf{R}) \cdot \frac{\partial \Psi(\mathbf{R})}{\partial \mathbf{R}} \quad \text { for } 0 \leqslant x \leqslant L,
\end{array}\right.
$$

given on the unperturbed waveguide surface $\mathbf{R}=\mathbf{R}_{\mathbf{s}}$, which is translationally invariant along the $x$ axis $[\mathbf{R}=(x, \mathbf{r})]$. The boundary condition (2) corresponds either to a waveguide surface with a random admittance $\boldsymbol{\xi}$, or to the Dirichlet boundary condition on a slightly perturbed waveguide surface, $\boldsymbol{\xi}$ denoting the random perturbation. In the latter case the boundary condition (2) is an approximate one, containing the first two terms in the expansion of the exact (Dirichlet) boundary condition about the unperturbed surface.

Outside the region $0 \leqslant x \leqslant L$ the solutions of the scattering problem under consideration have the form

$$
\begin{gathered}
\Psi_{n}(x, \mathbf{r})=\sum_{m} \frac{1}{\sqrt{k_{m}}} \chi_{m}(\mathbf{r}) e^{-i k_{m} x} t_{m n}, \quad x<0, \\
\Psi_{n}(x, \mathbf{r})=\frac{1}{\sqrt{k_{n}}} \chi_{n}(\mathbf{r}) e^{-i k_{n} x}+\sum_{m} \frac{1}{\sqrt{k_{m}}} \chi_{m}(\mathbf{r}) e^{i k_{m} x} r_{m n} \\
x>L
\end{gathered}
$$

The indexes $m, n$ correspond to the outgoing and incoming modes, respectively, and $\chi_{n}(\mathbf{r})$ are the eigenfunctions of the transverse wave equation

$$
\left(\frac{\partial^{2}}{\partial \mathbf{r}^{2}}+\kappa_{n}^{2}\right) \chi_{n}(\mathbf{r})=0
$$

with $k_{n}=\sqrt{k^{2}-\kappa_{n}^{2}}$.

By assuming that $\boldsymbol{\xi}=\boldsymbol{\xi} \mathbf{n}$, the boundary condition (2) can be also rewritten as

$$
\Psi\left(\mathbf{R}_{\mathbf{s}}\right)+\xi\left(\mathbf{R}_{\mathbf{s}}\right) \Phi\left(\mathbf{R}_{\mathbf{s}}\right)=0,
$$

where the normal derivative

$$
\Phi\left(\mathbf{R}_{\mathbf{s}}\right) \equiv \mathbf{n}\left(\mathbf{R}_{\mathbf{s}}\right) \cdot\left[\frac{\partial \Psi(\mathbf{R})}{\partial \mathbf{R}}\right]_{\mathbf{R}=\mathbf{R}_{\mathbf{s}}} \equiv \frac{\partial \Psi}{\partial n}
$$

is introduced, with $\mathbf{n}\left(\mathbf{R}_{\mathbf{s}}\right)$ being the normal to the unperturbed surface $\mathbf{R}=\mathbf{R}_{\mathrm{s}}$.

Let us employ Green's theorem in the form

$$
\begin{aligned}
\Psi(\mathbf{R})= & \int d^{3} R^{\prime} \frac{\partial}{\partial \mathbf{R}^{\prime}} \cdot\left[\Psi\left(\mathbf{R}^{\prime}\right) \frac{\partial G_{0}\left(\mathbf{R}^{\prime}, \mathbf{R}\right)}{\partial \mathbf{R}^{\prime}}\right. \\
& \left.-\frac{\partial \Psi\left(\mathbf{R}^{\prime}\right)}{\partial \mathbf{R}^{\prime}} G_{0}\left(\mathbf{R}^{\prime}, \mathbf{R}\right)\right],
\end{aligned}
$$

where the integral is taken over any region containing the point $\mathbf{R}$ and located inside the waveguide. The most convenient integration region is the unperturbed waveguide surface cut by two planes normal to the axis of the waveguide (they are included too). The left (right) cross section must be placed to the left (right) of the point $\mathbf{R}=(x, \mathbf{r})$. The integral in Eq. (7) can then be expressed as the sum of the integrals over the cross sections and the unperturbed waveguide surface with constraint $0 \leqslant x^{\prime} \leqslant L$ :

$$
\Psi(\mathbf{R})=\int d \mathbf{S}^{\prime} \cdot \Psi\left(\mathbf{R}_{\mathbf{s}}^{\prime}\right)\left[\frac{\partial G_{0}\left(\mathbf{R}^{\prime}, \mathbf{R}\right)}{\partial \mathbf{R}^{\prime}}\right]_{\mathbf{R}^{\prime}=\mathbf{R}_{\mathrm{s}}^{\prime}}+I_{\text {cross }} .
$$

The integral $I_{\text {cross }}$ over the cross sections may be easily calculated from Eqs. (3) and the Green's function 


$$
G_{0}\left(\mathbf{R}, \mathbf{R}^{\prime}\right)=\sum_{m=1}^{N} \frac{\chi_{m}(\mathbf{r}) \chi_{m}\left(\mathbf{r}^{\prime}\right)}{2 i k_{m}} e^{i k_{m}\left|x-x^{\prime}\right|} .
$$

Then Eq. (8) takes the form

$$
\begin{aligned}
\Psi_{n}(\mathbf{R})= & \frac{1}{\sqrt{k_{n}}} \chi_{n}(\mathbf{r}) e^{-i k_{n} x}+\int d \mathbf{S}^{\prime} \cdot \Psi_{n}\left(\mathbf{R}_{\mathbf{s}}^{\prime}\right) \\
& \times\left[\frac{\partial G_{0}\left(\mathbf{R}^{\prime}, \mathbf{R}\right)}{\partial \mathbf{R}^{\prime}}\right]_{\mathbf{R}^{\prime}=\mathbf{R}_{\mathbf{s}}^{\prime}} .
\end{aligned}
$$

The (oriented) surface element $d \mathbf{S}$ can be written as $d \mathbf{S}$ $=\mathbf{n} d S, d S=d x d s$. The explicit form of the differential $d s$ depends on the geometry under consideration $(d s=r d \phi$ for circular cross sections, $d y d z$ for rectangular cross sections, etc.). Then we can rewrite Eq. (10) as

$$
\begin{aligned}
\Psi_{n}(\mathbf{R})= & \frac{1}{\sqrt{k_{n}}} \chi_{n}(\mathbf{r}) e^{-i k_{n} x}+\int_{0}^{L} d x^{\prime} \oint d s^{\prime} \Psi_{n}\left(x^{\prime}, \mathbf{r}_{\mathbf{s}}^{\prime}\right) \\
& \times \frac{\partial G_{0}\left(x^{\prime}, x ; \mathbf{r}_{\mathbf{s}}^{\prime}, \mathbf{r}\right)}{\partial n^{\prime}} .
\end{aligned}
$$

Two conclusions can be derived from Eq. (11). First, the matrix of the reflection coefficients can be written [after substituting the explicit expression for $G_{0}$ from Eq. (9)] in the form

$$
r_{m n}=\frac{1}{2 i} \int_{0}^{L} d x \oint d s \frac{1}{\sqrt{k_{m}}} \phi_{m}(\mathbf{r}) e^{-i k_{m} x} \Psi_{n}\left(x, \mathbf{r}_{\mathbf{s}}\right),
$$

or [by the use of Eq. (5)] as

$$
r_{m n}=-\frac{1}{2 i} \int_{0}^{L} d x \oint d s \phi_{m}(x, s) \xi(x, s) \Phi_{n}(x, s),
$$

where

$$
\phi_{n}(x, s)=\frac{1}{\sqrt{k_{n}}} \mathbf{n}\left(\mathbf{r}_{\mathbf{s}}\right) \cdot\left[\frac{\partial \chi_{n}(\mathbf{r})}{\partial \mathbf{r}}\right]_{\mathbf{r}=\mathbf{r}_{\mathbf{s}}} e^{-i k_{n} x} .
$$

The second result we derive from Eq. (11) [by differentiating, setting $\mathbf{R}$ on the surface, and substituting the boundary condition (5)] is a closed equation for $\Phi_{n}(x, s)$ :

$$
\begin{aligned}
\Phi_{n}(x, s)= & \phi_{n}(x, s)-\int_{0}^{L} d x^{\prime} \oint d s^{\prime} \xi\left(x^{\prime}, s^{\prime}\right) \\
& \times \Phi_{n}\left(x^{\prime}, s^{\prime}\right) G_{0}^{\prime \prime}\left(x^{\prime}, x ; s^{\prime}, s\right) .
\end{aligned}
$$

Here $G_{0}^{\prime \prime}$ is the mixed normal derivative

$$
G_{0}^{\prime \prime}\left(x^{\prime}, x ; s^{\prime}, s\right)=\frac{\partial^{2} G_{0}}{\partial n \partial n^{\prime}}=\frac{1}{2 i} \sum \frac{1}{k_{n}} \frac{\partial \chi_{n}}{\partial n} \frac{\partial \chi_{n}}{\partial n^{\prime}} e^{i k_{n}\left|x-x^{\prime}\right|} .
$$

Differentiation of Eq. (14) yields

$$
\begin{aligned}
\frac{\partial \Phi_{n}(x, s)}{\partial L}= & \sum_{m} \phi_{m}(x, s) a_{m n}-\int_{0}^{L} d x^{\prime} \oint d s^{\prime} \xi\left(x^{\prime}, s^{\prime}\right) \\
& \times \frac{\partial \Phi_{n}\left(x^{\prime}, s^{\prime}\right)}{\partial L} G_{0}^{\prime \prime}\left(x^{\prime}, x ; s^{\prime}, s\right) \\
a_{m n}= & -\frac{1}{2 i} \oint d s^{\prime} \phi_{m}^{*}(L, s) \xi(L, s) \Phi_{n}(L, s)
\end{aligned}
$$

By comparing Eq. (15) with Eq. (14) we obtain the relation between the derivative $\partial \Phi_{n}(x, s) / \partial L$ and the functions $\left\{\Phi_{m}(x, s)\right\}$ :

$$
\frac{\partial \Phi_{n}(x, s)}{\partial L}=\sum_{m} \Phi_{m}(x, s) a_{m n} .
$$

With the aid of the latter equation, we now differentiate the matrix of reflection coefficients Eq. (13):

$$
\begin{aligned}
\frac{d r_{m n}}{d L}= & -\frac{1}{2 i} \oint d s \phi_{m}(L, s) \xi(L, s) \Phi_{n}(L, s) \\
& -\frac{1}{2 i} \int_{0}^{L} d x \oint d s \phi_{m}(x, s) \xi(x, s) \sum_{m} \Phi_{m}(x, s) a_{m n} .
\end{aligned}
$$

By substituting the explicit expressions for $\Phi_{m}(x, s)$ from Eqs. (3b) and (6), and collecting all terms, we arrive at

$$
\frac{d \hat{r}}{d L}=\frac{i}{2}\left(e^{-i \hat{k} L}+\hat{r} e^{i \hat{k} L}\right) \hat{v}\left(e^{-i \hat{k} L}+e^{i \hat{k} L} \hat{r}\right) .
$$

Here $\hat{k}=\operatorname{diag}\left(k_{n}\right)$ and

$$
\begin{gathered}
v_{m n}=\oint d s \phi_{m}(s) \xi(L, s) \phi_{n}(s), \\
\phi_{n}(s)=\frac{1}{\sqrt{k_{n}}}\left[\frac{\partial \chi_{n}(\mathbf{r})}{\partial \mathbf{r}}\right]_{\mathbf{r}=\mathbf{r}_{\mathbf{s}}} .
\end{gathered}
$$

Analogous algebra leads to the equation for the matrix of transmission coefficients:

$$
\frac{d \hat{t}}{d L}=\frac{i}{2} \hat{t} e^{i \hat{k} L} \hat{v}\left(e^{-i \hat{k} L}+e^{i \hat{k} L} \hat{r}\right)
$$

From the reflection and transmission amplitudes, we define the reflection and transmission intensities, respectively,

$$
R_{m n}=\left|r_{m n}\right|^{2}, \quad T_{m n}=\left|t_{m n}\right|^{2},
$$

which yield the intensity coupled into the $m$ th outgoing channel in reflection and transmission, respectively, for a given $n$th incoming channel. The reflectance and transmittance for the $n$th incident mode are

$$
R_{n}=\sum_{m} R_{n m}, \quad T_{n}=\sum_{m} T_{n m} .
$$

Finally, the total transmitted intensity in the case that all incoming channels are incoherently populated, which is equivalent to the dimensionless conductance for electrons, is 


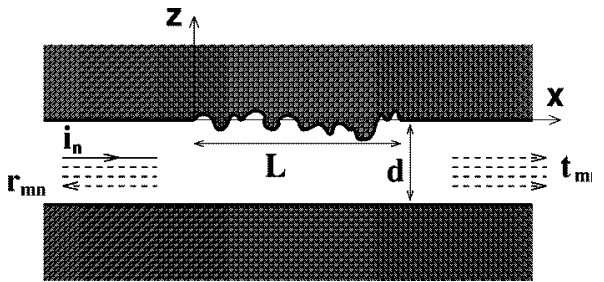

FIG. 1. Illustration of the waveguide geometry.

$$
g=\sum_{n} T_{n}
$$

\section{NUMERICAL CALCULATIONS}

For the numerical simulations we choose the simplest geometry (see Fig. 1): two parallel planes $z=0$ and $z=d$ with one-dimensional (1D) deviations $\xi=\xi(x)$ on one plane $(z$ $=0)$ only, where $\xi$ is a 1D stochastic process. Thus, the transverse eigenfunctions acquire the form

$$
\chi_{n}(z)=\sqrt{\frac{2}{d}} \sin \left(\kappa_{n} z\right), \quad \kappa_{n}=\frac{\pi n}{d}, \quad k_{n}=\sqrt{\left(\frac{\omega}{c}\right)^{2}-\kappa_{n}^{2}},
$$

and the impurity matrix becomes

$$
v_{m n}(L)=\frac{2}{d} \frac{\kappa_{n} \kappa_{m}}{\left(k_{n} k_{m}\right)^{1 / 2}} \xi(L) .
$$

The $2 N \times 2 N$ system of linear differential equations (19) is solved numerically by means of the sixth-order RungeKutta method. For each realization $\xi(x)$ (of length $L_{\max }$ ) of an ensemble of randomly rough surface profiles, the matrices of reflection and transmission amplitudes are calculated as functions of the length $L$. These realizations obey Gaussian statistics (with $\delta$ the rms height) with zero mean and a Gaussian correlation function

$$
W\left(\left|x-x^{\prime}\right|\right)=\delta^{-2}\left\langle\xi(x) \xi\left(x^{\prime}\right)\right\rangle=\exp \left[-a^{-2}\left(x-x^{\prime}\right)^{2}\right],
$$

where $a$ is the transverse correlation length. The corresponding surface-power spectrum is thus given by

$$
g(Q)=\pi^{1 / 2} a \exp \left[-(Q a)^{2} / 4\right] .
$$

The ensemble of surface realizations are numerically generated as described in Ref. 24. By averaging over $N_{p}$ such realizations, the mean values $\langle A\rangle$ and fluctuations $\delta A$ $=\left(\left\langle A^{2}\right\rangle-\langle A\rangle^{2}\right)^{1 / 2}$ of the relevant physical quantities are obtained. Hereafter we consider, unless otherwise stated, a waveguide of thickness $d=2.25 \lambda$ supporting $N=4$ guided modes.

\section{REFLECTION AND TRANSMISSION COEFFICIENTS}

In Fig. 2 the $\left\langle T_{m n}\right\rangle$ are shown (in a semilogarithmic scale) for $L \leqslant L_{\max }=1500 \lambda$. Averaging was carried out over the results obtained for $N_{p}=4000$ realizations of the surface profile, whose roughness parameters are $a=0.2 \lambda$ and $\delta$ $=0.03 \lambda$. The asymmetry in the behavior of the different outgoing channels $m$ is evident from this plot. The intensity

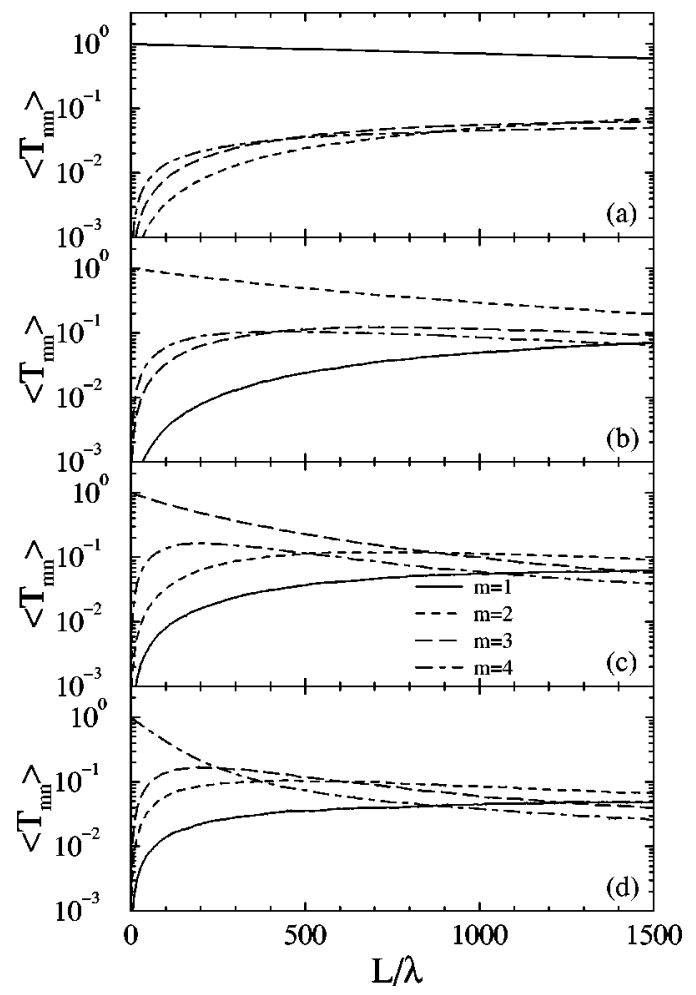

FIG. 2. Mean-transmission intensities $\left\langle T_{m n}\right\rangle$ as functions of length $L$ in semilogarithmic scale for a waveguide of width $d / \lambda$ $=2.25$, supporting four modes, with disorder parameters $a / \lambda=0.2$ and $\delta / \lambda=0.03$ : (a) incident mode $n=1$; (b) $n=2$; (c) $n=3$; (d) $n=4$. Averaged over $N_{p}=4000$ realizations.

of the incoming mode $\left\langle T_{n n}\right\rangle$ decreases with length for all $n$, this decrease being steeper the larger the transverse momentum $\kappa_{n}$, namely, the larger $n$. The transmission into other nondiagonal channels $m \neq n$ also depends strongly on the mode $m$. In the beginning of the waveguide, this nondiagonal transmission slightly increases from zero, being stronger into higher modes $m\left(\left\langle T_{m+1, n}\right\rangle>\left\langle T_{m n}\right\rangle\right.$, with $\left.m+1, m \neq n\right)$. In this situation only single scattering is important, and we refer to this regime as quasiballistic (QB). In accordance with the results of the perturbation theory (PT) the intensity of mode $m$ is proportional to the cross section for roughness-induced scattering from mode $n$ into mode $m$, and to the length $L$, as follows:

$$
\left\langle T_{m n}\right\rangle=\frac{2 \delta^{2} \kappa_{n} \kappa_{m}}{d^{2}\left(k_{n} k_{m}\right)^{1 / 2}} g\left(\left|k_{n}-k_{m}\right|\right) L \equiv \frac{L}{l_{m n}^{Q B}} .
$$

For the diagonal transmission, PT predicts

$$
\begin{aligned}
\left\langle T_{n n}\right\rangle & =1-L \frac{2 \delta^{2}}{d^{2}} \sum_{m=1}^{N} \frac{\kappa_{n} \kappa_{m}}{\left(k_{n} k_{m}\right)^{1 / 2}}\left[g\left(\left|k_{n}-k_{m}\right|\right)+g\left(\left|k_{n}+k_{m}\right|\right)\right] \\
& \equiv 1-\frac{L}{l_{n n}^{Q B}} .
\end{aligned}
$$

In Fig. 3 the corresponding QB lengths $l_{m n}^{Q B}$ from the preceding PT expressions have been plotted along with those obtained by fitting the numerical results shown in Fig. 2 to the expected linear functions, showing good agreement. 


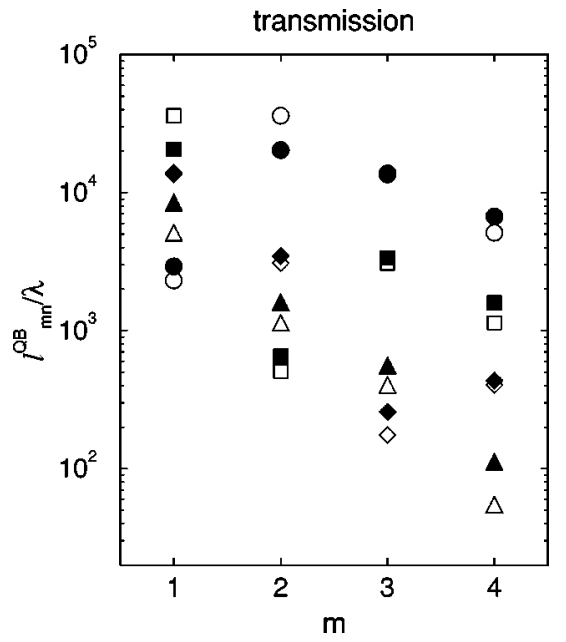

FIG. 3. QB lengths $l_{m n}^{Q B}$ (in wavelength units) in transmission vs outgoing channel $m$ for the waveguide used in Fig. 2: Circles, $n$ $=1$; squares, $n=2$; diamonds, $n=3$; triangles, $n=4$. Filled (open) symbols denote the numerical simulation (perturbation theory) results.

The origin of such asymmetries lies in the surface-type disorder that randomizes the wave propagation through the waveguide. If we look at the impurity matrix (24), which determines the scattering strength in Eqs. (19), it is obvious that there are large quantitative differences in $v_{m n}$ for distinct values of $m$ and $n$. As a matter of fact, this matrix can be rewritten as

$$
v_{m n}=\frac{8 d^{2}}{L^{2}} \frac{\xi(L)}{d}\left(k_{n} k_{m}\right)^{1 / 2} M_{m} M_{n},
$$

where $M_{n}$ is

$$
M_{n}=\frac{L \kappa_{n}}{2 d k_{n}} .
$$

Through a simple geometrical argument, as long as $\delta \ll d$, $M_{m}$ can be considered the number of times that mode $m$ hits (interacts with) the rough wall on its way along the waveguide. ${ }^{23}$ In the case that $d / \lambda=2.25$, it turns out that, for instance, $M_{4} \approx 8.5 M_{1}$. This factor affects the impurity matrix not only for the outgoing mode through $M_{m}$, but also for the incoming mode through $M_{n}$. This gives a physically intuitive explanation of the results shown in Fig. 2, and of all other processes that will be shown below.

For larger $L$ multiple scattering becomes relevant. This actually means that not only the scattering that brings energy to mode $m$ from $n$ should be taken into account, but also the leakage from $m$ into other modes, as well as all interchanges between $i$ and $j$ for all $i, j$. As a result, the energy spreads over all modes: diffusion (D) in the space of mode numbers takes place. ${ }^{25}$ In fact, it is seen in Fig. 2 that all outgoing channels tend to yield comparable transmission intensities within the length of the plot, except for $n=1$.

Furthermore, after a long propagation distance through the waveguide so that mode conversion has sufficiently populated all outgoing channels, we observe that $T_{1 n}>T_{2 n}$ $>T_{3 n}>T_{4 n}$. This waveguide length is not reached within the length scale covered in Fig. 2. Alternatively, we have

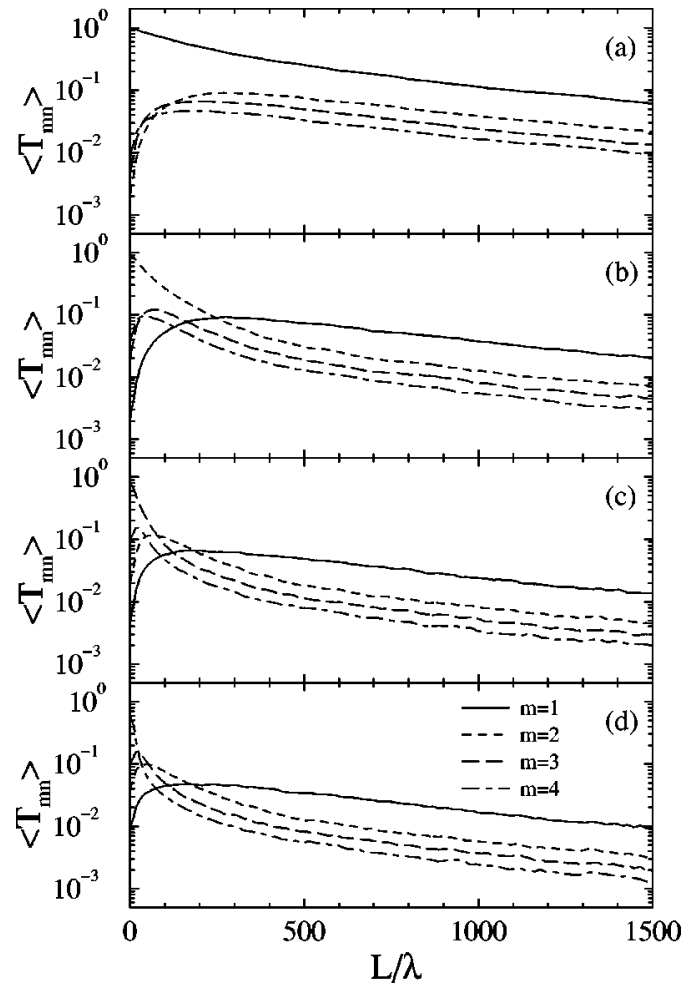

FIG. 4. Same as in Fig. 2 but for $\delta / \lambda=0.1$.

increased the surface roughness to $\delta=0.1 \lambda$, so that this result can be observed: the corresponding transmission coefficients, presented in Fig. 4, indeed confirm such behavior for a waveguide length $L \geqslant 300 \lambda$. Thus, the higher modes appear to be more strongly scattered. This is also manifested in the overall behavior of the four outgoing channels depending on the incident channel. Figure 4 reveals that, beyond the waveguide length given above, the transmission curves appear to be qualitatively similar for all incoming modes $n$, but shifted downward as $n$ is increased. The behavior of those transmission curves, following parallel exponential decays, is a signature of the onset of localization (L) due to the coherent interference of multiple-scattered waves. (On the other hand, it is interesting to note that, if we zoom in Fig. 4 for $0<L$ $<100 \lambda$, the transmission curves are qualitatively similar to those of Fig. 2.)

Thus, we have seen in Figs. 2 and 4 that the dependence of the impurity matrix (29) on mode dispersion has significant quantitative consequences, and also strong qualitative consequences for the properties of wave propagation through surface-disordered waveguides. As has been demonstrated in Ref. 23, it can give rise to an entangling of transport behaviors within the same waveguide length. In Fig. 5, the diagonal-transmission coefficients $T_{n n}$ from Fig. 4 are shown in a $\log$ plot. The results have been fitted, where possible, to the well-known behaviors: QB as in Eq. (28), inverse power law expected for D,

$$
\left\langle T_{n n}\right\rangle \approx \frac{l_{n n}^{D}}{L}
$$

and exponential decay associated with L. It is seen in Fig. 5 that, within the interval $10<L / \lambda<70$, QB transport of the (11) channel coexists with D for the (33) and (44) channels; 


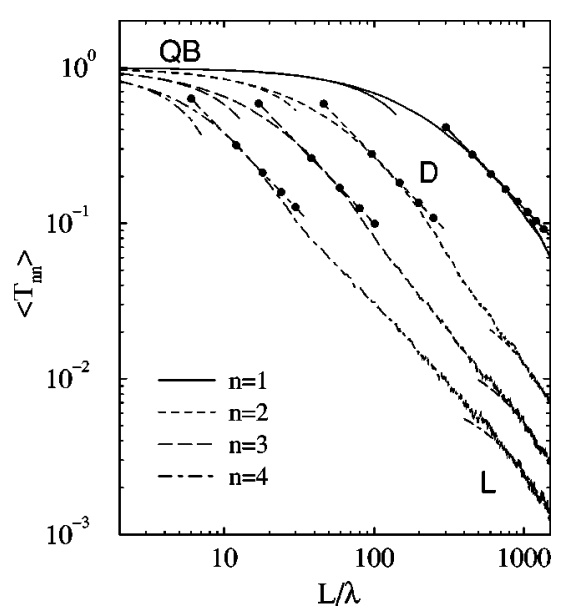

FIG. 5. Mean-diagonal transmission intensities $\left\langle T_{n n}\right\rangle$ as in Fig. 4 in a $\log$-log plot. Fits to linear (QB), $L^{-1}$ (D, with dots), and exponential (L) decays are shown.

also, D of the (11) mode coexists with $\mathrm{L}$ of the (44) channel for $L / \lambda \approx 10^{3}$. This confirms the coexistence of QB transport $\mathrm{D}$, and $\mathrm{L}$ predicted in Ref. 23 for eight-mode waveguides; nonetheless, in this four-mode waveguide the coexistence of all three regimes within the same length region is not observed due to the limitation in mode-dispersion differences associated with the lower number of available modes. [On the other hand, it should be noted that our results, not shown here, reveal such coexistence (QB-D or D-L) phenomena associated with surface-type disorder in the case of narrower waveguides supporting only three or even two guided modes.] Interestingly, the impossibility of defining the D regime consistently for all outgoing modes at the same length scale makes irrelevant any comparison with theories such as the macroscopic approach provided by the random-matrix theory (RMT), ${ }^{14,26}$ which predicts $\left\langle T_{m n}\right\rangle=\langle g\rangle / N^{2}$ for all $m, n$.

In addition, Fig. 5 permits us to observe the crossover between different regimes for each mode separately. For $L$ $<l_{44}^{Q B}$, all four modes propagate almost ballistically. The transition from ballistic transport to $\mathrm{D}$ can be observed for all modes at the distinct waveguide lengths defined by the corresponding $l_{n n}^{D}$ (see Fig. 6 below). Note that even though QB and $\mathrm{D}$ regimes extend over different $L$ regions, in both cases the regions are well defined by the magnitude of the transmission coefficient: $\left\langle T_{n n}\right\rangle \approx 1$ for $\mathrm{QB}$ and $\left\langle T_{n n}\right\rangle \approx 10^{-1}$ for $\mathrm{D}$. This seems to indicate that, from the value of the average transmission coefficient, the qualitative transport behavior can be roughly known, in agreement with Ref. 27, although there exist remarkable differences concerning the length dependence and the entangling of regimes. Finally, coherent interference leads to L. In Fig. 5 all modes [mode (11) barely] reach the $\mathrm{L}$ regime within the maximum length of the waveguide $L_{\max }$. It should be remarked that, whereas the exponential decay rate is similar for all $n$, the real onset of localization takes place at slightly different lengths: the lower $n$ is, the longer the waveguide must be to observe L.

The dependence of $l_{n n}^{Q B}, l_{n n}^{D}$, and $l_{n n}^{L}$ on surface roughness is shown in Fig. 6. In this respect, with the aim of correctly defining $l_{n n}^{L}$, the average of the logarithm of the transmission has been used: ${ }^{7}$

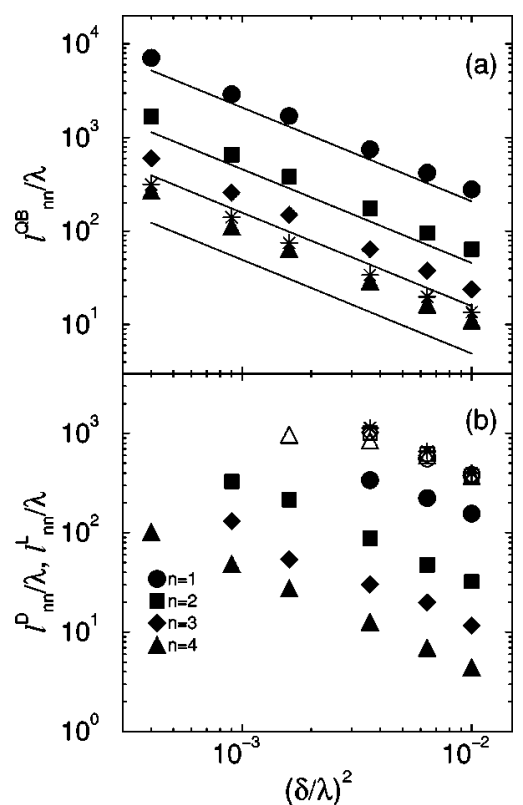

FIG. 6. Typical decay lengths (in wavelength units) as functions of the height standard deviation $\delta^{2}$ (in $\lambda^{2}$ units), obtained from the mean-transmission intensities (see text) for $d / \lambda=2.25$ and $a / \lambda$ $=0.2$. Circles, $n=1$; squares, $n=2$; diamonds, $n=3$; triangles, $n$ $=4$. (a) $l_{n n}^{Q B}$ from numerical simulation data (symbols, asterisks denoting the conductance $l^{Q B}$ ) and from perturbation theory (solid lines); (b) $l_{n n}^{D}$ (filled symbols) and $l_{n n}^{L}$ (open symbols), and $l^{L}$ from the conductance (asterisks).

$$
\left\langle\ln T_{n n}\right\rangle \approx-\frac{L}{l_{n n}^{L}} .
$$

The predicted $\delta^{-2}$ behavior is seen in Fig. 6(a) for the QB decay lengths, showing reasonable agreement with the PT results [cf. Eq. (28)]. Similar behavior is observed in Fig. 6(b) for $l_{n n}^{D}$ and $l_{n n}^{L}$. It is interesting to note that $l_{n n}^{D}$ is different for each $n$, whereas $l_{n n}^{L}$ coincides for all $n$. Thus, the well-known relationship $l^{L}=N l^{D}$ is meaningless in this context. (Although $l^{L}=N l_{11}^{D}$ seems to hold instead; in fact, it has been shown that if $l^{D}$ is defined through the resistance, $l^{L}$ $=N l^{D}$ does hold. ${ }^{28}$ )

The normalized fluctuations $\delta T_{m n} /\left\langle T_{m n}\right\rangle$ are shown in Fig. 7. It is evident that there are differences among the fluctuations for every channel, in agreement with the behavior of the mean values shown in Fig. 4; this corroborates the qualitative argument given above in connection with the asymmetry in the mode-scattering rates. Note that at the beginning of the waveguide, mode conversion into $m \neq n$ leads to a variance of unity for the corresponding off-diagonal fluctuations, whereas the diagonal $m=n$ ballistic transport is revealed through the result that $\left\langle\delta T_{n n}\right\rangle \approx 0$. Furthermore, these diagonal fluctuations undergo the crossovers between $\mathrm{QB}, \mathrm{D}$, and $\mathrm{L}$ regimes as discussed above in light of the mean values (see Fig. 5). The ( $n n)$ fluctuations exhibit an increase from 0 towards 1 as the transport gradually changes from QB to D. The well-known speckle-pattern fluctuations $\delta T_{n n} /\left\langle T_{n n}\right\rangle \approx 1$ for all $(m n)$ channels build up in the D regime, steadily increasing above 1 as the mode becomes localized. Therefore, the phenomenon of the QB-D and D-L coexistence can be recognized by comparing the diagonal 


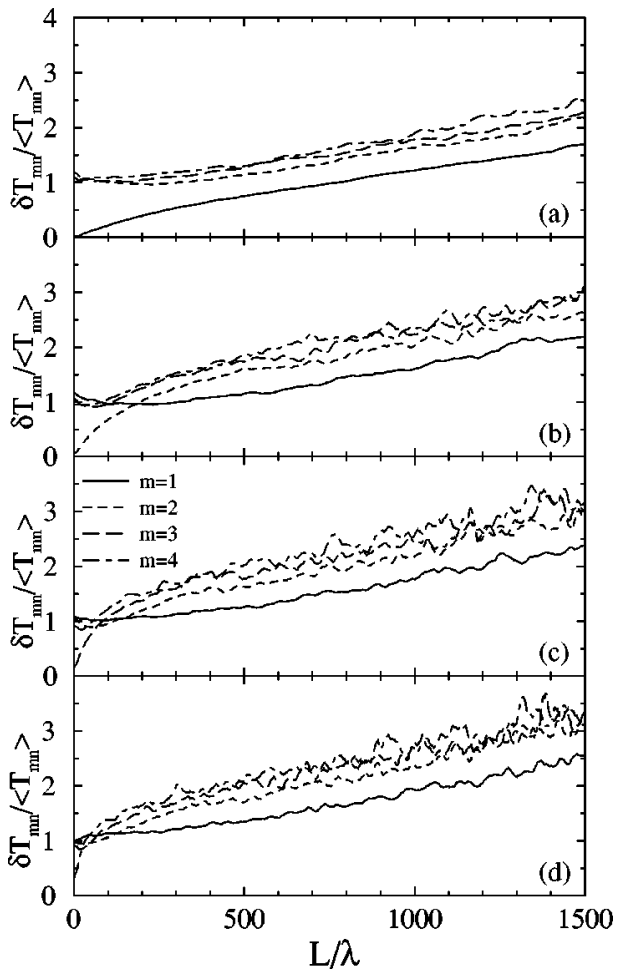

FIG. 7. Normalized fluctuations of the transmission intensities as functions of the length $L$, for the same parameters as in Fig. 4.

fluctuations with each other, corroborating the argument given above in light of the results for the mean values in Fig. 5. For a sufficiently long waveguide, it can be seen in Fig. 7 that the normalized fluctuations tend to be larger the higher the outgoing mode $m$ is. A linear increase for all channels is observed. ${ }^{10}$ For a given incoming mode $n$, the rate of increase is the same for all outgoing channels $m$; nonetheless, the fluctuations appear to be larger the higher $m$ is (within the noise accuracy). Analogously, the rate of increase is faster the higher $n$ is. These considerations corroborate the arguments discussed above on the mode selectivity of the scattering strength in connection with the transmission intensities in Fig. 4.

Let us now turn to the study of the reflection coefficients $\left\langle R_{m n}\right\rangle$. These are presented in Fig. 8 for the same waveguide considered in Fig. 4. The peculiar scattering properties induced by surface disorder manifest themselves in an intricate manner in the reflection channels also. For sufficiently short waveguide lengths, we expect that the reflection coefficients should increase linearly as predicted by PT, through the expressions:

$$
\left\langle R_{m n}\right\rangle=\frac{2 \delta^{2} \kappa_{n} \kappa_{m}}{d^{2}\left(k_{n} k_{m}\right)^{1 / 2}} g\left(\left|k_{n}+k_{m}\right|\right) L \equiv \frac{L}{l_{m n}^{Q B}} .
$$

These PT QB decay lengths in reflection and those obtained from the numerical results are shown in Fig. 9. The agreement is even better than in transmission, and the strong mode differences are indeed confirmed. Beyond the QB regime for each incoming mode $n$, the diagonal $\left\langle R_{n n}\right\rangle$ is enhanced as the waveguide length increases, whereas the remaining offdiagonal reflection coefficients exhibit differences with the following tendency: the higher is the mode $m$, the larger is

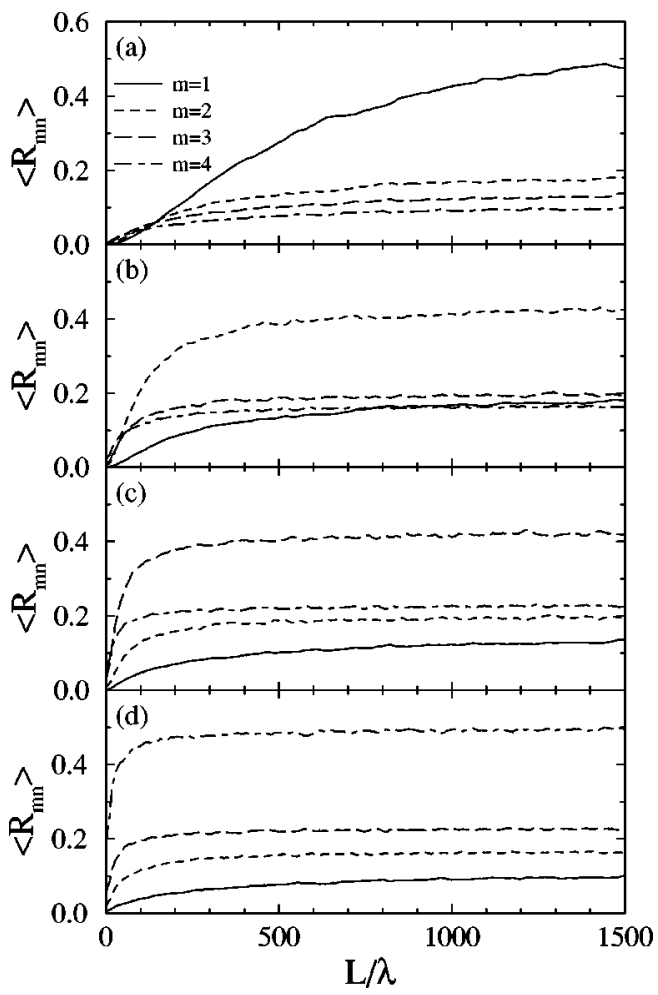

FIG. 8. Same as Fig. 4 but for the mean-reflection intensities $\left\langle R_{m n}\right\rangle$.

the reflection coefficient. Enhanced backscattering appears in the strong diffusive (or weak localization) regime as a result of the constructive interference of multiple-scattered paths; nonetheless, the enhancement factor differs from 2 (predicted by simple arguments, provided that the single-scattering contribution is absent or negligible). This comes as no surprise inasmuch as each channel may behave differently, as revealed in the transmission coefficients (see Fig. 4) through the entangling of transport regimes. Interestingly enough, this anomalous reflection can result in an anomalous enhancement factor, ${ }^{29}$ larger than 2 [see Fig. 8(a): although the background cannot be unambiguously defined, $\left\langle R_{11}\right\rangle$ $\approx 2.3\left\langle R_{m 1}\right\rangle$ for any $\left.m \neq 1\right]$. Therefore, the reflection coefficients fail to satisfy ${ }^{14,26}\left\langle R_{m n}\right\rangle=\left(1+\delta_{m n}\right) N^{-1}(1+N)^{-1}(N$

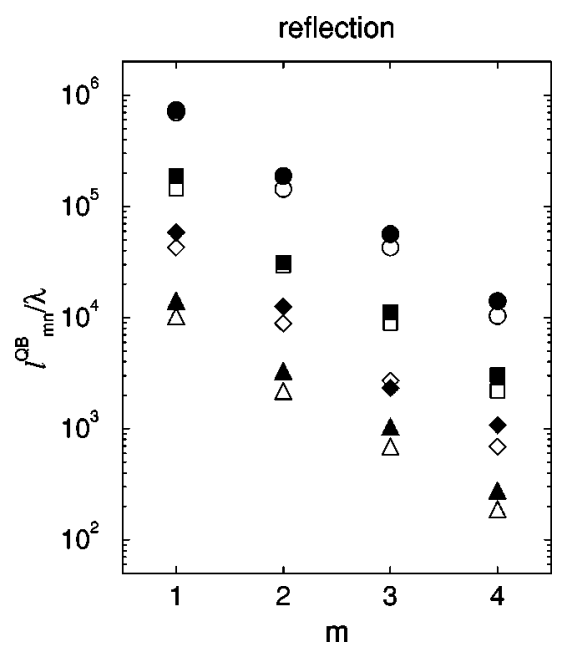

FIG. 9. Same as Fig. 3 but in reflection. 


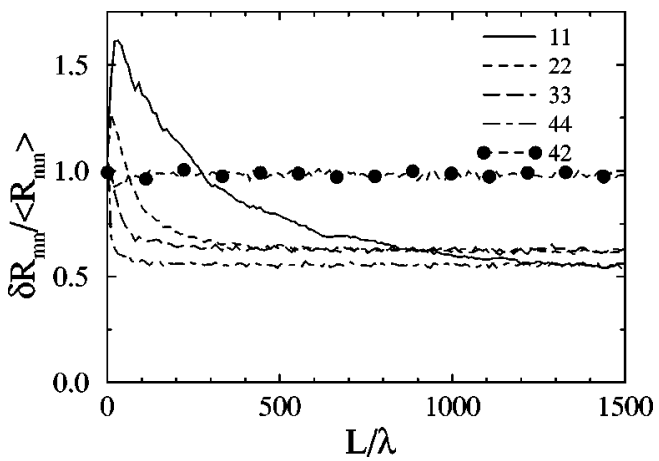

FIG. 10. Same as Fig. 7 but for the normalized fluctuations of the reflection intensities, including only the backscattered channels and the (42) off-diagonal channel.

$-\langle g\rangle)$. Figure 8 also seems to indicate that the onset of $\mathrm{L}$ does not introduce significant changes in the reflection coefficients, in agreement with Ref. 29.

It is interesting to analyze the normalized fluctuations of the reflection coefficients (see Fig. 10). Leaving aside the transient strong fluctuations for very short length scales (associated with the fact that the corresponding reflection coefficients are small), the diagonal $m=n$ normalized fluctuations diminish with increasing length, this decrease being steeper the higher $n$ is. Then they stabilize about the value $\delta R_{n n} /\left\langle R_{n n}\right\rangle=0.5$ as the $\mathrm{D}$ regime is reached, and remain constant when entering into the $\mathrm{L}$ regime. The off-diagonal normalized fluctuations [only the (42) channel is shown in Fig. 10, since all the rest are similar], on the other hand, remain about the variance of unity linked to the specklepattern fluctuations in reflection. Therefore, weak localization halves speckle pattern fluctuations in backscattering.

\section{REFLECTANCE, TRANSMITTANCE, AND CONDUCTANCE}

We have thus seen that wave propagation along a fourmode surface-disordered waveguide, due to the surface-type disorder, unlike for volume disorder, displays anomalous properties in the transmission and reflection coefficients as a consequence of the mixture of $\mathrm{QB}, \mathrm{D}$, and $\mathrm{L}$ regimes for different waveguide channels. Bearing in mind these properties, we now proceed to calculate the total transmission $T_{n}$, reflection $R_{n}$, and dimensionless conductance $g$.

In Fig. 11, we plot the mean total reflection $\left\langle R_{n}\right\rangle$ and transmission $\left\langle T_{n}\right\rangle$ coefficients, along with the transmission fluctuations $\delta T_{n}$, in our four-mode waveguide with $\delta$ $=0.03 \lambda$. It is evident that these quantities differ substantially from one incoming mode to another. The larger $n$ is, the larger the mean reflectance and the smaller the mean transmittance (recall that energy conservation requires that $R_{n}$ $+T_{n}=1$ ). This could be qualitatively expected once again, at least in the limit of small waveguide lengths, in light of the $n$ dependence of the impurity matrix (29), which is stronger for incoming modes with larger transverse momentum $\kappa_{n}$ (higher $n$ ).

The overall transport properties of the waveguide for a given incoming mode $n$ can be understood through the behavior of the mean total transmission $\left\langle T_{n}\right\rangle$ [see Fig. 11(b)], as the summation of $\left\langle T_{m n}\right\rangle$ over all outgoing channels $m$.

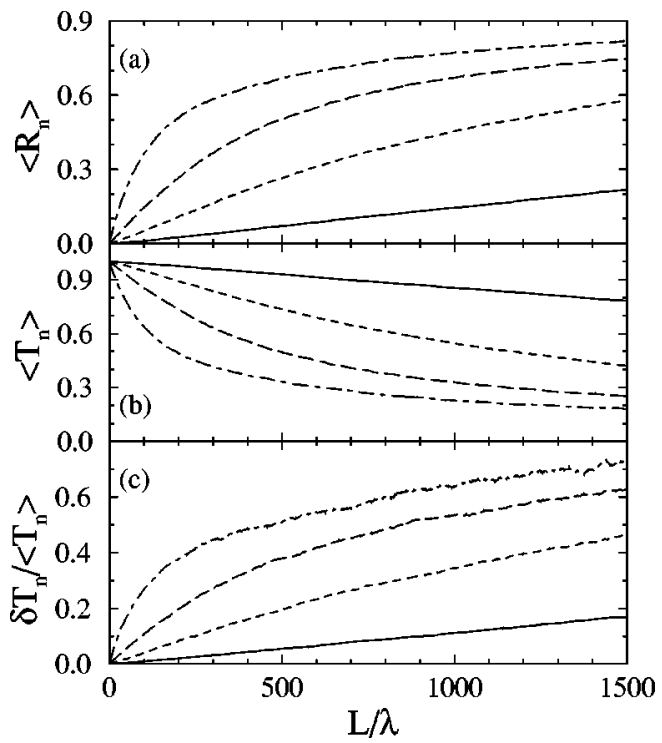

FIG. 11. Mean total (a) reflection and (b) transmission intensities, and (c) normalized fluctuations of the total transmission intensities, as functions of the length $L$, for the same parameters as in Fig. 2.

Figure 12, which shows $\left\langle T_{n}\right\rangle$ in a logarithmic plot for a four-mode waveguide analogous to that of Fig. 11(b) but with a rougher surface with $\delta=0.1 \lambda$, illustrates this discussion. To observe a definite transport regime in the total transmission, either the transport regimes of the different outgoing modes swap at certain length scales, or one of the $\left\langle T_{m n}\right\rangle$ 's predominates over all others. Note that even though the most transparent mode gives the predominant contribution from a quantitative standpoint, it is not at all evident that the same is true for the qualitative behavior (for instance, a steeper, weak decay added to a larger, but smoother, background would yield as a result a quantity whose magnitude is of the order of the latter, but whose qualitative behavior is given by the former weak decay). In principle, it can be assessed that transport will obviously be QB for lengths shorter than $l_{n n}^{Q B}$, namely, for $L \leqslant l_{n n}^{Q B}$, as Fig. 12 reveals through the QB linear decays (see also Fig. 5); this can also

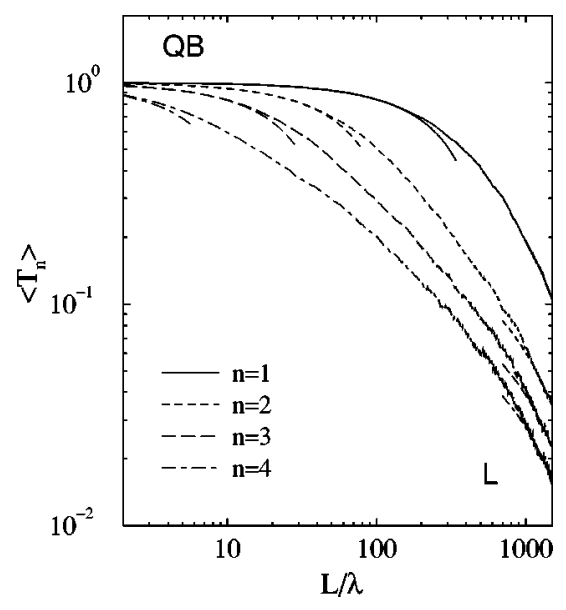

FIG. 12. Mean total-transmission intensities $\left\langle T_{n}\right\rangle$ as functions of the length $L$ in a $\log -\log$ plot for the same parameters as in Fig. 4, including fits to the $\mathrm{QB}$ and $\mathrm{L}$ regimes. 


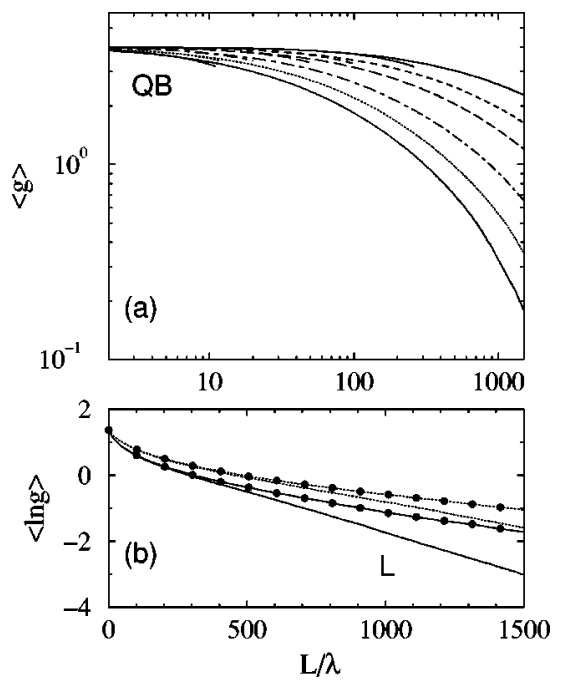

FIG. 13. (a) Mean dimensionless conductance as a function of the length $L$ in a log-log plot for a waveguide width $d / \lambda=2.25$, supporting four modes, with disorder parameters $a / \lambda=0.2$ and $\delta / \lambda=0.02$ (upper solid curve), 0.03 (dashed curve), 0.04 (long dashed curve), 0.06 (dot-dashed curve), 0.08 (dotted curve), and 0.1 (lower solid curve). Averages over $N_{p}=4000$ realizations. Fits to the QB regimes are shown. (b) $\langle\ln g\rangle$ (without dots) and $\ln \langle g\rangle$ (with dots) for $\delta / \lambda=0.08$ (dotted curves) and 0.1 (solid curves), revealing the $\mathrm{L}$ regimes.

be verified in Fig. 11(b). Conversely, the exponential decay associated with $\mathrm{L}$ appears beyond waveguide lengths for which the lowest $(1 n)$ mode is localized $l_{1 n}^{L}$, as seen in Fig. 12 (see also Figs. 4 and 5). Finally, unlike the QB and L regimes, which must always be encountered for sufficiently small and large lengths, respectively, it is not obvious that the $L^{-1}$ dependence (D regime) in the intermediate region between QB and L transport is observed. This effect is another manifestation of the entangling of different transport regimes of the $\left\langle T_{m n}\right\rangle$ due to the combination of surface-type disorder and large mode dispersion. In Fig. 12, where the length dependence of $\left\langle T_{n}\right\rangle$ is shown in a log scale, D should manifest itself through a linear decay [cf. Eq. (31)]. It is seen that this decay is practically absent for most incoming modes. Only within a narrow waveguide length window for which the D length scales of the transmission coefficients swap, would the corresponding total transmission exhibit the expected $L^{-1}$ behavior. In any case, it is obvious that the average reflection and transmission coefficients fail to obey the predicted dependences $\left\langle T_{n}\right\rangle=N^{-1}\langle g\rangle$ and $\left\langle R_{n}\right\rangle$ $=N^{-1}(N-\langle g\rangle)$ in the weak localization or D regime. ${ }^{14,26}$

All these transmission properties, stemming from the mixing of $\mathrm{QB}, \mathrm{D}$, and $\mathrm{L}$ transports produced by surface disorder, become even more pronounced in the dimensionless conductance $g$. Figure 13(a) shows a logarithmic plot of $\langle g\rangle$ for four-mode waveguides with different surface roughness parameters $\delta / \lambda=0.02,0.03,0.04,0.06,0.08$, and 0.1 , whereas $\langle\ln g\rangle$ is plotted in Fig. 13(b) [and also $\ln \langle g\rangle$ ] for the two larger $\delta$ values. The corresponding conductance fluctuations are given in Fig. 14. Following the argument mentioned above for the total transmission, now the QB regime is restricted to the shorter $l_{n n}^{Q B}$, in this case $l_{44}^{Q B}$ [see Fig. 13(a)]. This is explicitly shown in Fig. 6(a). Likewise, the true L behavior in the conductance is ensured for lengths beyond

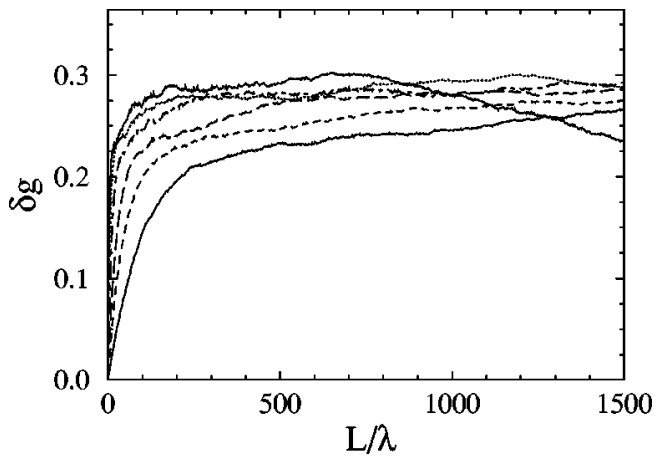

FIG. 14. Conductance fluctuations for the waveguides used in Fig. 13.

which the (11) channel appears localized; this is seen in Fig. 13(b) (in those cases for which $l^{L} \leqslant L_{\max }$ ) through the linear decay of $\langle\ln g\rangle$, and its departure from $\ln \langle g\rangle$ (owing to the transition to log-normal statistics, for which the dominant contributions arise from the low-probability realizations that yield large conductances ${ }^{7,11}$ ). Recall that, although the actual onset of L thus appears at slightly different lengths, the localization length for given roughness parameters as defined from Eq. (32) is the same for all modes $(m n)$, and coincides with those for the transmittances and conductance [see Fig. $6(b)]$. On the other hand, the absence of the D regime in the conductance curves is explicitly displayed in Fig. 13(a). Thus an anomalous conductance crossover from QB to L regimes is observed for the four-mode, surface-disordered waveguides with several roughness parameters used in obtaining the results of Fig. 13, giving additional confirmation of the predictions of Ref. 23. Moreover, the conductance fluctuations within this anomalous transition regime stabilize in all cases shown in Fig. 14 at a value $(\delta g \approx 0.29)$, which lies below the expected value of the quasi-1D universal conductance fluctuations (UCF) for a well-defined D regime ( $\delta g \approx 0.364$, cf. Refs. 10 and 12 ). This lower value of the UCF has been also numerically found in Ref. 11, but no physical interpretation was given therein. When entering into the $L$ regime, our results for the waveguides with rougher surfaces in Fig. 14 reveal that the conductance fluctuations decrease below the UCF region, as expected. ${ }^{10,11}$

Two comments are in order concerning the anomalous QB-L crossover in the total transmission and conductance mentioned above. First, it should be emphasized that surface disorder is not a sufficient condition. Actually, in the case of surface-disordered waveguides with small mode dispersion and/or strong intermode mixing, so that the D-like regimes of different outgoing channels coexist, the $L^{-1}$ diffusive dependence could also be observed. Nevertheless, even if such a $\mathrm{D}$ regime appears, our results still reveal an anomalous behavior, inasmuch as the mean total reflection and transmission fail to follow the predicted weak-localization length dependences, as pointed out above. As a second remark, it is worth mentioning that the D-like regime is enhanced in the average resistance (in which contributions from smaller transmission coefficients predominate), in contrast to the average conductance. The results presented in Ref. 28 corroborate these comments, which thus show no discrepancy with our results. 


\section{CONCLUSIONS}

The statistical-transport properties of classical waves propagating along surface-disordered waveguides have been studied, with special emphasis on the distinctive imprint introduced by the surface-type disorder. For this purpose, the invariant embedding equations for the matrices of reflection and transmission amplitudes of the guided modes have been obtained. By means of Monte Carlo simulation calculations, in such a manner that for every surface realization the corresponding system of coupled differential equations is numerically solved, the statistical quantities of interest are calculated. We have focused on the mean reflection and transmission coefficients, reflectances, transmittances, and conductance, along with their fluctuations. The interplay between mode conversion and the scattering processes produced by surface disorder results in new interesting effects in the physical quantities mentioned.

For a sufficiently long waveguide, transmission into modes with lower transverse momentum (lower index $m$ according to our notation) is favored (exhibiting smaller normalized fluctuations), no matter what the incoming mode $n$ is. The influence of the incoming mode $n$ is revealed in the decrease of all the transmission coefficients for higher $n$. For smaller waveguide lengths, we have confirmed, through the analysis of the length dependence of the mean-transmission coefficients and fluctuations in the case of four-mode waveguides, the entangling of ballistic, diffusive, and localized transmission of modes within the same waveguide region that was recently reported in Ref. 23 in the case of eight-mode waveguides.

With regard to the reflection coefficients, enhanced backscattering is observed when the rough waveguide is long enough, and the enhancement factor, as defined by the ratio $\min \left[\left\langle R_{n n}\right\rangle /\left\langle R_{m n}\right\rangle\right]$, can be larger than 2 . In fact, the nondiagonal reflection coefficients tend to be smaller for the reflected modes with lower $m$ for all incoming modes $n$. The reflected speckle patterns exhibit reduced fluctuations in backscattering $\left(\delta R_{n n}\right) /\left\langle R_{n n}\right\rangle \approx 0.5$, whereas the expected value of 1 is approximately obtained for other reflected channels $(m$ $\neq n$ ). Both averages and fluctuations behave similarly throughout the $\mathrm{D}$ and $\mathrm{L}$ regimes.

The transmittance, namely, the normalized total energy transmitted for a given incoming mode $n$, is larger for the lower modes $n$. It should be noted that, in spite of the small strength of the random component that is present on one of the waveguide planes, very strong reflectances (of the order of or larger than $90 \%$ ) can be observed for sufficiently long waveguides. This could be relevant in multimode, optical waveguides with spuriously rough boundaries over long propagation distances, where it constitutes an unwanted effect. $^{30}$
We have also analyzed the effect of the entangling of QB, $\mathrm{D}$, and $\mathrm{L}$ transport on the qualitative behavior of the mean transmittance and its fluctuations, showing an anomalous effective QB-L crossover. This has also been confirmed in the conductance calculations (average and UCF), for which the influence of the disorder strength has been shown.

Finally, we would like to mention the very recent papers by García-Martín et al. on the diffusion-localization transition $^{28}$ and on the intensity distributions, ${ }^{27,29}$ in nanowires with surface-disordered hard walls consisting of a number of slices with fixed length and random width, with similarities to the problem dealt with here. Their numerical results, based on a generalized scattering-matrix formulation exploiting mode matching at each slice, exhibit also the nonisotropy of the scattering intensities, stressing, however, the agreement of the statistics at each transport regime with the RMT predictions.

Experimentally, all these effects can be revealed in the transmission intensities through metal microwave guides, for which our theoretical boundary conditions apply very accurately. As pointed out in Ref. 23, the appropriate geometry would be a planar waveguide with two metallic plates, one of them at least randomly rough, with feasible dimensions and roughness parameters (as derived from the values used throughout this paper upon scaling them by the wavelength in the centimeter range). Similar waveguides but with tube geometry have been successfully employed in connection with volume disorder. ${ }^{20}$ Also, in the electromagnetic domain, optical waveguides or fibers (in the micron range) could be other experimental devices, ${ }^{30}$ accessible to such measurements, where the predictions of our calculations can manifest themselves, although in order to make rigorous quantitative comparisons the boundary conditions might have to be revised. Furthermore, the propagation of acoustic waves or other classical waves through confined geometries with appropriate randomness can be adequately accounted for by our formulation, and thus similar phenomena might be expected therein. The conductance calculations can be also of interest in the electronic transport through nanowires.

\section{ACKNOWLEDGMENTS}

J.A.S.G. is grateful to A. García-Martín, J. A. Torres, J. J. Sáenz, and M. Nieto-Vesperinas for valuable discussions, and acknowledges support from the Spanish CSIC, DGES Grant No. PB97-1221 and CICYT Grant No. TIC95-0563CO5-03. The work of A.A.M. was supported in part by Army Research Office Grant No. DAAH 04-96-1-0187. I.V.Y. gratefully acknowledges support by EPSRC Grant No. GR/K95505.
${ }^{1}$ K. B. Efetov, Supersymmetry in Disorder and Chaos (Cambridge University Press, Cambridge, England, 1997).

${ }^{2}$ K. B. Efetov, Adv. Phys. 32, 53 (1984).

${ }^{3}$ B. A. Muzykantskii and D. E. Khmelnitskii, Pis'ma Zh. Éksp. Teor. Fiz. 62, 68 (1995) [JETP Lett. 62, 76 (1995)].
${ }^{4}$ V. Tripathi and D. E. Khmelnitskii, Phys. Rev. B 58, 1122 (1998).

${ }^{5}$ Ya. M. Blanter, A. D. Mirlin, and B. A. Muzykantskii, Phys. Rev. Lett. 80, 4161 (1998).

${ }^{6}$ Scattering and Localization of Classical Waves in Random Me- 
dia, edited by P. Sheng (World Scientific, Singapore, 1990).

${ }^{7}$ V. Freilikher and S. Gredeskul, Prog. Opt. 30, 137 (1992).

${ }^{8} \mathrm{P}$. Sheng, Introduction to Wave Scattering, Localization and Mesoscopic Phenomena (Academic, San Diego, 1995).

${ }^{9}$ P. A. Lee and T. V. Ramakrishnam, Rev. Mod. Phys. 57, 287 (1985); Mesoscopic Phenomena in Solids, edited by B. L. Altshuler, P. A. Lee, and R. A. Webb (North-Holland, Amsterdam, 1991).

${ }^{10}$ H. Tamura and T. Ando, Phys. Rev. B 44, 1792 (1991); T. Ando and H. Tamura, ibid. 46, 2332 (1992).

${ }^{11}$ K. Nikolić and A. MacKinnon, Phys. Rev. B 50, 11008 (1994).

${ }^{12}$ P. García-Mochales, P. A. Serena, N. García, and J. L. CostaKrämer, Phys. Rev. B 53, 10268 (1996); P. García-Mochales and P. A. Serena, Phys. Rev. Lett. 79, 2316 (1997).

${ }^{13}$ J. A. Torres and J. J. Sáenz, Phys. Rev. Lett. 77, 2245 (1996).

${ }^{14}$ C. W. J. Beenakker, Rev. Mod. Phys. 69, 731 (1997).

${ }^{15}$ I. Edrei, M. Kaveh, and B. Shapiro, Phys. Rev. Lett. 62, 2120 (1989).

${ }^{16}$ J. F. de Boer, M. C. W. van Rossum, M. P. van Albada, Th. M. Nieuwenhuizen, and A. Lagendijk, Phys. Rev. Lett. 73, 2567 (1994).

${ }^{17}$ Th. M. Nieuwenhuizen and M. C. W. van Rossum, Phys. Rev. Lett. 74, 2674 (1995).

${ }^{18}$ E. Kogan and M. Kaveh, Phys. Rev. B 52, R3813 (1995).

${ }^{19}$ S. A. van Langen, P. W. Brouwer, and C. W. J. Beenakker, Phys. Rev. E 53, 1344 (1997).
${ }^{20}$ M. Stoytchev and A. Z. Genack, Phys. Rev. Lett. 79, 309 (1997).

${ }^{21}$ P. W. Brouwer, Phys. Rev. B 57, 10526 (1997).

${ }^{22}$ N. Makarov and I. Yurkevich, Zh. Éksp. Teor. Fiz. 96, 1106 (1989) [Sov. Phys. JETP 69, 628 (1989)]; A. Krokhin, N. Makarov, V. Yampolskii, and I. Yurkevich, Physica B 165\&166, 855 (1990); V. Freilikher, M. Pustilnik, and I. Yurkevich, Phys. Rev. Lett. 73, 810 (1994).

${ }^{23}$ J. A. Sánchez-Gil, V. Freilikher, I. Yurkevich, and A. A. Maradudin, Phys. Rev. Lett. 80, 948 (1998).

${ }^{24}$ J. A. Sánchez-Gil and M. Nieto-Vesperinas, J. Opt. Soc. Am. A 8, 1270 (1991); Phys. Rev. B 45, 8623 (1992).

${ }^{25}$ F. Bass, V. Freilikher, and I. Fuks, IEEE Trans. Antennas Propag. 22, 288 (1974).

${ }^{26}$ K. A. Muttalib, J.-L. Pichard, and A. D. Stone, Phys. Rev. Lett. 59, 2475 (1987); P. A. Mello, E. Akkermans, and B. Shapiro, ibid. 61, 459 (1988); E. Bascones, M. J. Calderón, D. Castelo, T. López, and J. J. Sáenz, Phys. Rev. B 55, R11 911 (1997).

${ }^{27}$ A. García-Martín, J. A. Torres, J. J. Sáenz, and M. NietoVesperinas, Phys. Rev. Lett. 80, 4165 (1998).

${ }^{28}$ A. García-Martín, J. A. Torres, J. J. Sáenz, and M. NietoVesperinas, Appl. Phys. Lett. 71, 1912 (1997).

${ }^{29}$ A. García-Martín, T. López-Ciudad, J. J. Sáenz, and M. NietoVesperinas, Phys. Rev. Lett. 81, 329 (1998).

${ }^{30}$ F. Ladouceur and L. Poladian, Opt. Lett. 21, 1833 (1996). 